\title{
Inhaltsübersicht
}

Seite

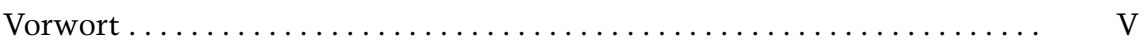

Aus dem Vorwort zur 2. Auflage $2009 \ldots \ldots \ldots \ldots \ldots \ldots \ldots \ldots \ldots \ldots$ V

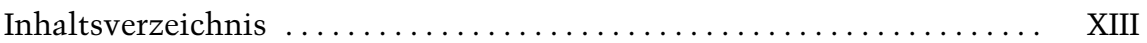

Abkürzungsverzeichnis ............................. XXXIII

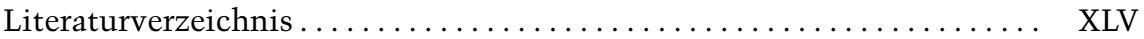

\section{Erster Teil:}

\section{Einführung in das System des kollektiven Arbeitsrechts}

$\S 75$ Begriff des kollektiven Arbeitsrechts $\ldots \ldots \ldots \ldots \ldots \ldots \ldots \ldots \ldots$

$\S 76$ Zweck des kollektiven Arbeitsrechts ................... 2

$\S 77$ Bedeutung des kollektiven Arbeitsrechts ................. 3

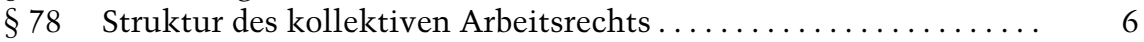

$\S 79$ Literatur zum kollektiven Arbeitsrecht ................. 9

\section{Zweiter Teil:}

\section{Das Recht der Koalitionen}

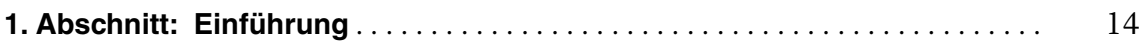

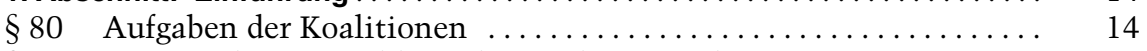

$\S 81$ Historische Entwicklung des Koalitionsrechts ............... 15

2. Abschnitt: Koalitionsbegriff und Koalitionsfreiheit $\ldots \ldots \ldots \ldots \ldots \ldots . \ldots 17$

$\S 82$ Der Koalitionsbegriff ............................ 17

$\S 83$ Der Schutzbereich der Koalitionsfreiheit nach Art. 9 Abs. 3 GG . 30

$\S 84$ Grenzen der Koalitionsfreiheit . . . . . . . . . . . . . . . . . . . . 49

3. Abschnitt: Aufbau und Organisation der Koalitionen .............. 72

$\S 85$ Das Industrieverbandsprinzip . . . . . . . . . . . . . . . . . . . . . 72

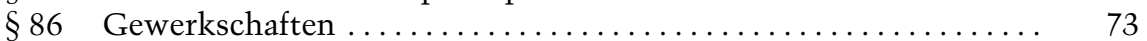

$\S 87$ Arbeitgeberverbände ............................. 75

\section{Dritter Teil:}

\section{Tarifvertragsrecht}

1. Abschnitt: Abschluss des Tarifvertrags . . . . . . . . . . . . . . . . . 77

$\S 88$ Funktionen des Tarifvertrags ........................ 77

$\S 89$ Zustandekommen des Tarifvertrags $\ldots \ldots \ldots \ldots \ldots \ldots \ldots \ldots . \ldots . \ldots . \ldots 1$

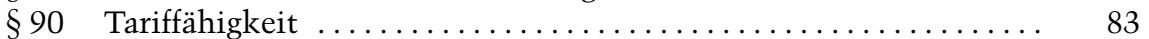

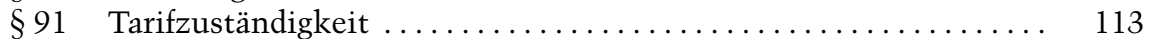

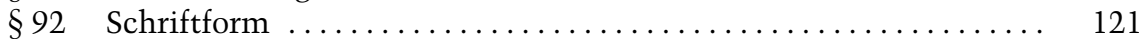

$\S 93$ Bekanntgabe des Tarifvertrags ...................... 122 


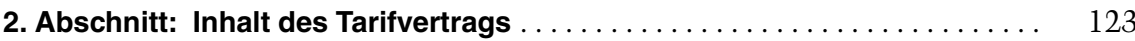

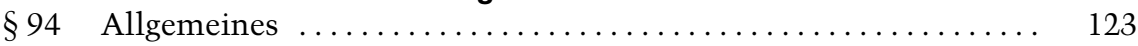

$\S 95$ Normativer Teil ................................. 130

$\S 96$ Schuldrechtlicher Teil ............................. 140

$\S 97$ Auslegung von Tarifverträgen ..................... 147

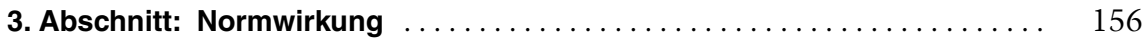

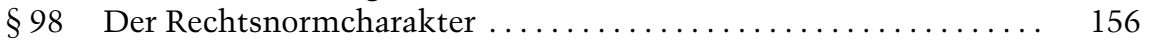

4. Abschnitt: Tarifgebundenheit und Geltungsbereich … . . . . . . . . 195

$\S 99$ Tarifgebundenheit ................................. 195

$\S 100$ Geltungsbereich ................................. 237

$\S 101$ Tarifkonkurrenz und Tarifpluralität $\ldots \ldots \ldots \ldots \ldots \ldots \ldots \ldots \ldots . \ldots \ldots$

5. Abschnitt: Rechtsnatur des Tarifvertrags/Grenzen der Regelungsmacht . 270

$\S 102$ Rechtsnatur des Tarifvertrags ..................... 270

$\S 103$ Grundlage der Normsetzungsbefugnis ................. 270

$\S 104$ Verhältnis der Tarifnormen zu anderen Rechtsquellen ......... 275

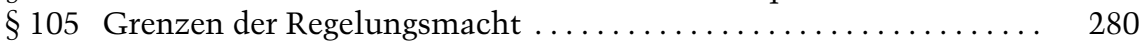

6. Abschnitt: Internationales Tarifvertragsrecht $\ldots \ldots \ldots \ldots \ldots \ldots \ldots \ldots$

$\S 106$ Überstaatliche Tarifverträge ......................... 314

$\S 107$ Tarifrecht mit Auslandsberührung .................. 317

\section{Vierter Teil:}

\section{Arbeitskampf und Schlichtungswesen}

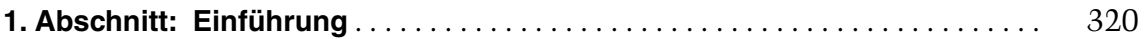

$\S 108$ Grundgedanken und Grundlagen des Arbeitskampfrechts . . . . . . 321

$\S 109$ Rechtsgrundlage des Arbeitskampfs ... . . . . . . . . . . . . . . . . 329

$\S 110$ Ausgestaltung des Arbeitskampfrechts durch Gesetz und Richter-

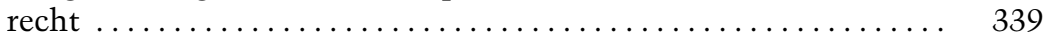

$\S 111$ Grundsätze des Arbeitskampfrechts .................. 344

2. Abschnitt: Begriff und Mittel des Arbeitskampfs … . . . . . . . . . . 352

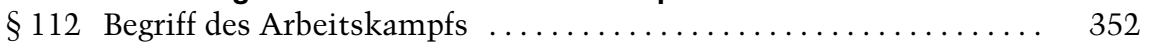

$\S 113$ Arbeitskampfmittel ............................... 354

3. Abschnitt: Rechtmäßigkeitsvoraussetzungen eines Arbeitskampfs .... 365

$\S 114$ Zulässiges Arbeitskampfziel . ..................... 366

$\S 115$ Anforderungen an die Kampfparteien .................. 378

$\S 116$ Voraussetzungen für den Kampfbeginn .................... . 389

$\S 117$ Verhältnismäßigkeitsgrundsatz . . . . . . . . . . . . . . . 392

$\S 118$ Wahrung der Rechtsordnung als Schranke des Arbeitskampfs . . . 412

4. Abschnitt: Zur Vertiefung: Rechtmäßigkeit besonderer Arbeitskampfmittel und -taktiken . . . . . . . . . . . . . . . . . . . . . . . . . . . . 413

$\S 119$ Wellenstreik ................................... 414

$\S 120$ Betriebsblockade und -besetzung; Flashmob ................ 416

$\S 121$ Schlechtleistung und partielle Arbeitsniederlegung . . . . . . . . . . 421

$\S 122$ Suspendierende Betriebsstilllegung ................... 422 
$\S 123$ Streikbruchprämie .......................... 424

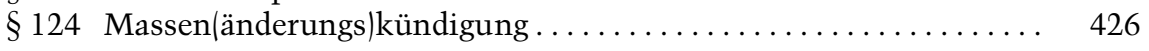

5. Abschnitt: Rechtsfolgen rechtmäßiger Arbeitskämpfe . . . . . . . . . . . 427

$\S 125$ Suspendierung der arbeitsvertraglichen Pflichten ............ 428

$\S 126$ Lösende Wirkung . . . . . . . . . . . . . . . . . . . . . . 433

$\S 127$ Anderweitige Rechtsfolgen ......................... 434

6. Abschnitt: Rechtsfolgen rechtswidriger Arbeitskämpfe . . . . . . . . . . . 437

$\S 128$ Rechtsfolgen eines rechtswidrigen Streiks ................. 438

$\S 129$ Rechtsfolgen einer rechtswidrigen Aussperrung . . . . . . . . . . . . . . 444

$\S 130$ Sozialrechtliche Auswirkungen .................... 446

7. Abschnitt: Rechtsfolgen für nicht unmittelbar beteiligte Dritte . . . . . . . 447

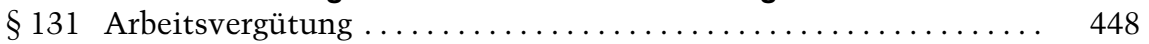

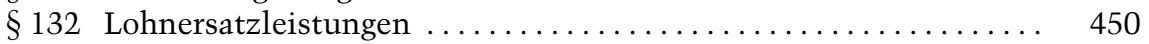

$\S 133$ Einführung von Kurzarbeit $\ldots \ldots \ldots \ldots \ldots \ldots \ldots \ldots \ldots \ldots \ldots \ldots . \ldots \ldots \ldots \ldots \ldots \ldots \ldots$

8. Abschnitt: Arbeitskampfstreitigkeiten .................... 451

$\S 134$ Allgemeine Voraussetzungen ....................... 452

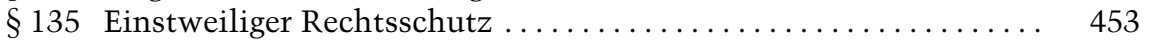

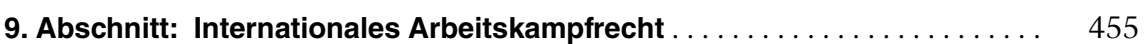

$\S 136$ Arbeitskampfstatut ........................... 456

$\S 137$ Beispiele für Arbeitskämpfe mit Auslandsbezug . . . . . . . . . . . . . 457

$\S 138$ Sonderproblem: Streiks mit Europarechtsbezug . . . . . . . . . 458

10. Abschnitt: Tarifliches Schlichtungsrecht $\ldots \ldots \ldots \ldots \ldots \ldots \ldots \ldots \ldots$

$\S 139$ Grundlagen des Schlichtungsrechts .................... 463

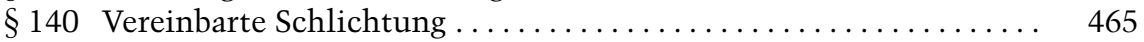

$\S 141$ Staatliche Schlichtung ........................... 467

\section{Fünfter Teil:}

\section{Mitbestimmungsrecht}

1. Abschnitt: Einführung in das System des Mitbestimmungsrechts . . . . . . 469

$\S 142$ Grundlagen der Mitbestimmung ...................... 469

$\S 143$ System der Mitbestimmung im deutschen Arbeitsrecht . . . . . . . 473

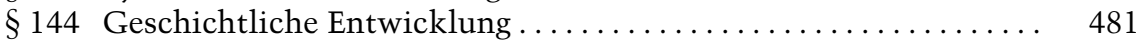

2. Abschnitt: Betriebsverfassungsrecht $\ldots \ldots \ldots \ldots \ldots \ldots \ldots \ldots \ldots \ldots$

$\S 145$ Gliederung des Betriebsverfassungsrechts ............... . 487

$\S 146$ Leitprinzipien des Betriebsverfassungsgesetzes ............. 496

$\S 147$ Geltungsbereich und Zuständigkeitsabgrenzungen des Betriebsverfassungsrechts ............................ 509

$\S 148$ Wahl, Organisation und Rechtsstellung des Betriebsrats ....... 571

$\S 149$ Rechtsstellung der Koalitionen . . . . . . . . . . . . . . . . . . . . . . 619

$\S 150$ Rechtsstellung der Arbeitnehmer .................... 624

$\S 151$ Beteiligungsrechte des Betriebsrats ................... 627

$\S 152$ Instrumente der gemeinsamen Entscheidungstätigkeit . . . . . . . 649

$\S 153$ Mitbestimmung in sozialen Angelegenheiten .............. 689 
$\S 154$ Gestaltung von Arbeitsplatz, Arbeitsablauf und Arbeitsumgebung 751

$\S 155$ Mitbestimmung in personellen Angelegenheiten .......... 753

$\S 156$ Mitbestimmung in wirtschaftlichen Angelegenheiten ........ 811

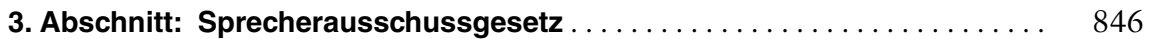

$\S 157$ Grundlagen der Sprecherverfassung . . . . . . . . . . . . . . . . . 846

$\S 158$ Organisation der Sprecherverfassung . . . . . . . . . . . . . . . . 849

$\S 159$ Mitwirkung der leitenden Angestellten ..................... 854

4. Abschnitt: Personalvertretungsrecht . . . . . . . . . . . . . . . . . . 858

$\S 160$ Grundlagen des Personalvertretungsrechts . . . . . . . . . . . . . . . 858

$\S 161$ Organisation der Personalvertretung ................... 860

$\S 162$ Beteiligungsrechte im Personalvertretungsrecht ............ 861

5. Abschnitt: Einführung in die Grundstruktur der Mitbestimmung auf Un-

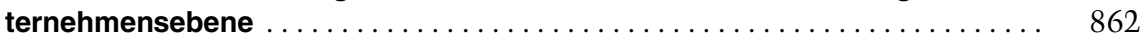

$\S 163$ Grundlagen ............................. 863

$\S 164$ Beteiligung der Arbeitnehmer an unternehmerischen Entschei-

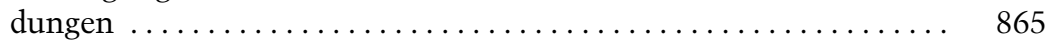

6. Abschnitt: Mitbestimmungsgesetz $1976 \ldots \ldots \ldots \ldots \ldots$. . . . . . . . . . . 869

$\S 165$ Geltungsbereich ............................... 869

$\S 166$ Mitbestimmung in Aufsichtsrat und Unternehmensleitung . . . . . 871

$\S 167$ Gegenstand der Mitbestimmung im Aufsichtsrat ........... 874

7. Abschnitt: Drittelbeteiligungsgesetz $2004 \ldots \ldots \ldots \ldots \ldots \ldots$. . . . . . . . 875

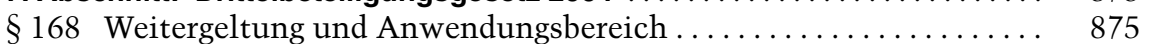

$\S 169$ Mitbestimmung nur im Aufsichtsrat .................. 876

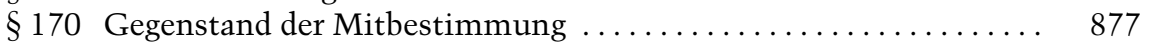

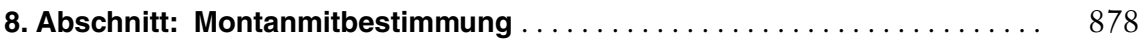

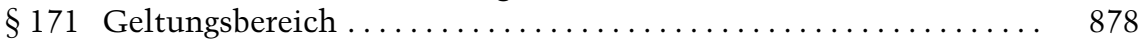

$\S 172$ Mitbestimmung in Aufsichtsrat und Unternehmensleitung . . . . . 879

$\S 173$ Gegenstand der Mitbestimmung im Aufsichtsrat ............ 881

9. Abschnitt: Mitbestimmung in grenzüberschreitenden Unternehmen und

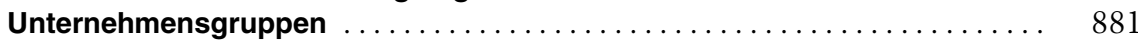

$\S 174$ Europäischer Betriebsrat ........................ 881

$\S 175$ Mitbestimmung in der Europäischen Gesellschaft (Societas Europaea, SE) .................................. 890

\section{Sechster Teil:} Arbeitsgerichtliches Verfahren

1. Abschnitt: Die Arbeitsgerichtsbarkeit . . . . . . . . . . . . . . . . . 899

$\S 176$ Funktionen und Besonderheiten ..................... 899

2. Abschnitt: Das Urteilsverfahren . . . . . . . . . . . . . . . . . . . . . . 907

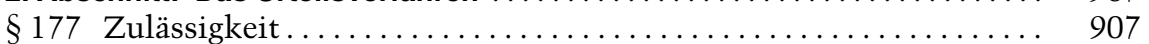

$\S 178$ Verfahrensablauf und Verfahrensbeendigung ............. 931 
Inhaltsübersicht

3. Abschnitt: Die Rechtsmittel im Urteilsverfahren . . . . . . . . . . . . . . . . . 940

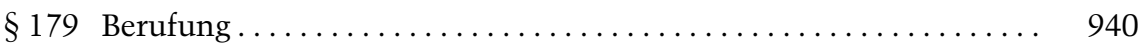

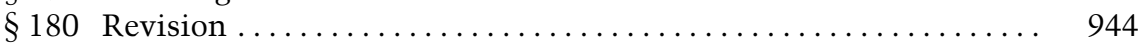

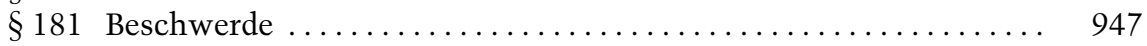

4. Abschnitt: Das Beschlussverfahren . . . . . . . . . . . . . . . . . . 949

$\S 182$ Zulässigkeit............................... 949

$\S 183$ Verfahrensablauf und Verfahrensbeendigung .............. 955

$\S 184$ Rechtsmittel ............................... 958

Stichwortverzeichnis . . . . . . . . . . . . . . . . . . . . . . . . . . . . 959 
